\title{
Effect of oral functional training on immunological abilities of older people: a case control study
}

\author{
Mitsue Sato ${ }^{1,2,3^{*}}$, Masahiro Sugimoto ${ }^{3,4,5}$, Yuko Yamamoto ${ }^{3}$, Juri Saruta ${ }^{3,6}$ and Keiichi Tsukinoki, ${ }^{3,6}$
}

\begin{abstract}
Background: Oral functional ability decreases with age, and systemic immunological ability and quality of life can also deteriorate. Continuous moderate whole-body exercise for older people is known to improve oral functional and their immunological abilities. Here, we evaluated the effect of oral exercise as an alternative training method for highly older people who cannot perform whole-body exercises.

Methods: Unstimulated whole saliva samples had been collected for three times before training as baseline data and one time after 3 and 6 weeks of training each. Participants were instructed to conduct self-massage; their tongues were used to press their orbicularis oris muscle and buccinators, and instructed to perform bilateral massage of three major glands for facilitating saliva secretion. Medical histories, daily life habits and characteristics were also collected.

Results: Totally 30 participants ( $84.2 \pm 8.5$ years) were enrolled. In contrast to previous researches, increase in salivary Immunoglobulin A (IgA) after the training was not observed. Interestingly, hierarchical clustering analyses revealed clear individual variations as two prominent clusters and a strong positive correlation between stimulated saliva flow rate and IgA flow rate, regardless of the continuous oral functional exercise. Only body mass index (BMI) showed significant differences between the two groups $(Z=2.06, P=0.039$, Wilcoxon rank-sum test) among all collected parameters.

Conclusion: Oral functional training limitedly effects on salivary parameters of highly older people. On the other hand, BMI characterized salivary features more than any other parameters, such as the presence of diseases or medication use in these people. Trial registration: UMIN-CTR Clinical Trial UMIN000028394 on 27/July 2017, retrospectively registered.
\end{abstract}

Keywords: Immunoglobulin a, "Aged, 80 and over", Saliva, Salivary gland, Exercise therapy

\section{Background}

Older people are at high risk of infectious diseases due to reduction of immune system function, and the average age of onset of reduced immune system function has reportedly begun to decrease [1-3]. Improving immune system function in aging people is expected to contribute to improving their overall health status. Therefore, the effects of various systemic exercises on immune system in elder people have been investigated [4-7].

\footnotetext{
* Correspondence: mitsue.sato@tohto.ac.jp

${ }^{1}$ Nursing Science, Ryotokuji University, Urayasu, Chiba 279-8567, Japan

${ }^{2}$ Nursing Science, Human Care Department, Tohto College of Health

Sciences, Fukaya, Saitama 366-0052, Japan

Full list of author information is available at the end of the article
}

Oral cavity plays an important role in various functions, including respiration and other essential biological functions, including eating and swallowing, along with articulation, which contribute to quality of life, systemic health and communication [8]. It is well known the association between worse oral health in elderly subjects and higher respiratory infection, such as pneumonia and influenza virus infection, including upper-respiratory tract infections (URTI) $[9,10]$. The associations between oral inflammatory disorders, such as gingivitis and periodontitis, and frailty and several diseases, atherosclerosis cardiovascular diseases, arthritis, and diabetes in older people [11]. Weakened oral cavity function, such as 
tooth loss, and lower oral hygiene in elder subjects reduces immune system function, and leads to decreased systemic health [12, 13]. Thus, many attempts of continuous oral care for elderly subjects to improve their systematic health have been reported $[9,14-16]$.

Although many evidences have been reported for the contribution of systemic exercise to improve immune function of elder people [4, 7], more moderate training or exercise is needed for them who cannot perform systemic exercise. Oral functional training, e.g. stimulating salivary grands and oral diadochokinesis [17], showed nutritional improvement [18] and also improvement of feeding function of elderly subjects [19]. Swallowing exercise showed improvement of salivary parameters, such as saliva flow rate, salivary $\mathrm{pH}$ at rest, and buffering capacity, suggesting the improvement of oral functions [20]. Thus, oral training is expected as an alternative exercise, which potentially contribute to immune functions of these elder people.

Indicators to objectively evaluate the effect of this training should be explored. Salivary secretory Immunoglobulin A (IgA) plays a central role in immune function in the oral cavity because it is a major effector of mucosal immunity by preventing submucosal invasion of pathogens [21]. Decreased secretory IgA secretion with aging is considered to be an indicator of attenuated immune system function, and leads to an increased incidence of respiratory tract infection [22]. There have been reports on recovery programs for attenuated immune systems comprising treatments such as oral functional exercise [23] and a combination of physical training and intake of lactobacilli [24]; however, data for highly older people ( $\geq 80$ years) are not available.

The aim of this study was to investigate the effect of oral functional exercise on the immune function in older people who were over the age of 80 years. Stimulated saliva flow rate and salivary IgA were used as indicators as immune functions. Oral functional exercise were administrated for totally 6 weeks and the change of these parameters were observed.

\section{Methods}

\section{Participants}

This study was retrospectively registered as a University hospital Medical Information Network Clinical Trials Registry (UMIN-CTR) Clinical Trial (Unique trial Number: UMIN000028394) on 27/July 2017. This study was approved by the ethics committee at Ryotokuji University (No. 2506) and Oasis Geriatric Health Services facilities. The research was performed, following to the Declaration of Helsinki in 1995 (as revised in Brazil 2013). Informed consent was obtained from the volunteer participants and their families. All participants were recruited consecutively from Oasis Geriatric Health
Services facilities. Subjects without any acute diseases, showing distinct consciousness and communication ability, were enrolled. The study was conducted from July to September 2013. Information on participant characteristics, dietary habits, diseases and medications was collected. Participants were recruited consecutively and subjects with xerostomia were excluded in this study. Written informed consent was obtained from each subject before participating in the study.

\section{Study protocol}

Participants were enrolled in oral functional exercises before every meal (3 times per day) under the supervision of nurses. Participants were required to conduct self-massage; their tongues were used to press their orbicularis oris muscle and buccinators. These training were conducted within 15 min. For facilitating saliva secretion, participants were instructed to perform bilateral massage of three major glands, as described below (slightly modified from refs. [23, 25]):

- Parotid glands: four fingers from the forefinger to the little finger are placed on the cheeks and the upper molar area is moved back and forth (10 times).

- Sublingual glands: push up slowly the bottom area of chin using the thumbs of both hands (10 to 20 times).

- Submandibular glands: the inside soft part of the jaw is pressed with the thumbs, from below the ear to the tip of the chin, and sequentially pressed in four to five areas (5 times).

Subsequently, tongue and mouth exercises were conducted to simulate the orbicularis oris muscle and the mimetic muscles [23], as follows:

- the tongue is held out as far as possible and moved horizontally left and right (10 times).

- the tongue is held out as far as possible and moved vertically up and down to lick the nose and chin (10 times).

- plosive sounds ("Pa", “Ta", "Ka" "Ra”) were intensively pronounced in a loud voice up to 10 times.

The first oral exercise was conducted at 7:30-7:15 a.m. and breakfast was served at 7:30-8:00 a.m. Saliva samples were collected at 9:30-10:00 a.m., to eliminate diurnal variation, just after the mouth was rinsed out twice with natural water, and also to eliminate the acute stress caused by the exercise. The second oral exercise was conducted at 11:30-11:45 a.m. and lunch was served at 12:00-12:30 p.m. The third oral exercise was conducted at 11:30-11:45 a.m. and an evening meal was served at 12:00-12:30 p.m. 
As baseline data, saliva samples were collected three times on different days when the oral functional exercise was not started, for the assessment of the daily variance of salivary parameters. The oral functional exercise were conducted totally 6 weeks. After 3 weeks of the oral functional exercise, saliva samples were collected. Subsequent 3 weeks of the oral functional exercise, saliva samples were collected again. Unstimulated whole saliva were collected using a sterilized cotton swab (Salikids; Saersted, Vümbrecht, Germany) for 2 min. We selected this product because of a string was attached to prevent from swallowing of the swab. The cotton was centrifuged at $626 \times \mathrm{g}$ and $4^{\circ} \mathrm{C}$ for $15 \mathrm{~min}$ and the concentration of IgA was quantified using an enzyme-linked immunosorbent assay (ELISA). Stimulated saliva secretion flow rate $(\mathrm{ml} / \mathrm{min})$ was calculated based on the quantified saliva volume divided by $2 \mathrm{~min}$, and the IgA secretion rate $(\mathrm{ml} / \mathrm{min})$ was calculated with the salivary secretion flow rate and IgA concentration.

\section{Measurement of salivary $\lg \mathrm{A}$}

IgA concentration in the saliva samples was quantified using a human IgA ELISA Quantitation Kit (Bethyl Laboratories, Montgomery, TX). Ninety-six-well microtitre plates were coated for $1 \mathrm{~h}$ at room temperature $\left(20-25{ }^{\circ} \mathrm{C}\right)$ with goat anti-human IgA (1:100 dilution) diluted with the coating buffer, $0.05 \mathrm{M}$ carbonate-bicarbonate, at $\mathrm{pH}$ 9.6. The residual fluid was removed and washed five times with wash solution (50 mM Tris, $0.14 \mathrm{M} \mathrm{NaCl}, 0.05 \%$ Tween 20, pH 8.0). Blocking solution $(50 \mathrm{mM}$ Tris, $0.14 \mathrm{M} \mathrm{NaCl}, 1 \%$ bovine serum albumin $\mathrm{pH}$ 8.0) was added to the wells as a blocking agent. Then the plate was incubated for $30 \mathrm{~min}$ at room temperature and washed with wash solution as described. Saliva samples and IgA standards (Bethyl Laboratories) were added to each well. The plate was incubated for $1 \mathrm{~h}$ at room temperature and washed five times with wash solution. Horseradish peroxidase-conjugated goat anti-human IgA detection antibody (1:75,000 dilution) was added to each well. The plate was incubated again for $1 \mathrm{~h}$ at room temperature. The enzyme substrate TMB was added to each well after washing. The plate was developed in the dark at room temperature for $15 \mathrm{~min}$. The reaction was stopped with $0.18 \mathrm{M} \mathrm{H}_{2} \mathrm{SO}_{4}$ stop solution. Absorbance was measured at a wavelength of $450 \mathrm{~nm}$ in an automated microplate reader (BioRad, Hercules, CA). Absolute concentrations $(\mu \mathrm{g} / \mathrm{ml})$ were calculated using a standard curve. The IgA secretion rate $(\mu \mathrm{g} / \mathrm{min})$ was calculated by multiplying the absolute concentration of IgA by stimulated saliva flow rate $(\mathrm{ml} / \mathrm{min})$.

\section{Data analysis}

The averaged value of three saliva samples was used as the baseline data for each individual. Standard deviation (SD) and relative standard deviation (RSD), i.e. SD divided by the average, was also calculated. Salivary volume and IgA concentration with these flow rates before and after oral functional exercise were compared using the Wilcoxon matched-pairs signed rank test (two-tailed). Hierarchical clustering using Euclidian distance was used to extract individual-specific patterns of these data. The differences in each feature of prominent clusters were accessed and corrected by false discovery rate (FDR), accommodating multiple independent tests. The Wilcoxon rank-sum test (two-tailed) and the $\mathrm{x} 2$ test (two-sided) were used for statistical analyses. $P<0.05$ was considered statistically significant. JMP (ver. 12.0.1, SAS Institute, Cary, NC), MeV TM4 [26], R (ver 3.2.3, R-Foundation for Statistical Computation, Vienna, Austria) and GraphPad Prism (ver 5.04, GraphPad Software Inc., San Diego, CA) were also used.

\section{Results}

\section{Salivary parameters}

Overall, 30 participants whose age was $84.2 \pm 8.5$ years (mean $\pm \mathrm{SD}$ ) were enrolled into this study. Linearity of IgA quantification was $R=0.99 \pm 0.029$ (Pearson correlation, $n=3$ ) using eight different concentrations between $0 \mu \mathrm{g} / \mathrm{ml}$ to $0.5 \mu \mathrm{g} / \mathrm{ml}$. RSD of the salivary secretion volume and IgA baseline concentration was $16.1 \%$ and $35.6 \%$, respectively. Data showing large differences (larger than 2-fold SD from the average) were treated as outliers and eliminated from the comparison between the data collected before and after exercise. Salivary secretion volume $(n=25)$ and $\operatorname{IgA}$ concentration $(n=27)$ data were used for statistical analyses. For the salivary secretion flow, data from participants who showed outlier data for either salivary secretion volume or IgA concentration were eliminated. Therefore, participants $(n=$ 23) whose age was $83.4 \pm 7.5$ years were used for the analysis of salivary secretion flow. Stimulated saliva flow rate, IgA concentration and IgA secretion rate are summarized in Table 1. Wilcoxon matched-pairs signed rank test was used here. There was no significant difference between the stimulated show saliva flow rate at baseline compared to after 3 weeks of exercise $(Z$-value $=-1.06$, $P=0.29$ ). In contrast, stimulated saliva flow rate after 6 weeks showed a significant decrease $(Z$-value $=-2.14$, $P=0.034)$. IgA concentration was significantly decreased after 3 weeks $(Z$-value $=-2.12, P=0.0035)$, while there was no significant difference after 6 weeks $(Z$-value $=$ -1.39, $P=0.17)$. IgA flow rate was significantly decreased after 3 weeks $(Z$-value $=-2.40, P=0.017)$ and 6 weeks $(Z$-value $=-2.13, P=0.035$; Fig. 1$)$.

\section{Clustering analysis}

Clustering analysis was conducted to understand the individual variation in the patterns of saliva and IgA flow of each participant, and it revealed three prominent clusters, indicating three representative patterns (Fig. 2). 
Table 1 Salivary parameters

\begin{tabular}{|c|c|c|c|c|c|c|c|c|c|c|c|c|c|}
\hline \multirow[t]{2}{*}{ Parameter } & \multirow[t]{2}{*}{$\mathrm{n}$} & \multicolumn{2}{|l|}{ Baseline } & \multicolumn{5}{|c|}{ After 3 weeks } & \multicolumn{5}{|c|}{ After 6 weeks } \\
\hline & & Average & SD & Average & SD & Z-Value & $P$-value & & Average & SD & Z-Value & $P$-value & \\
\hline Stimulate saliva flow rate $(\mathrm{ml} / \mathrm{min})$ & 25 & 0.563 & 0.185 & 0.515 & 0.168 & -1.06 & 0.29 & & 0.472 & 0.160 & -2.14 & 0.034 & $*$ \\
\hline IgA concentration $(\mu \mathrm{g} / \mathrm{ml})$ & 27 & 91.23 & 58.42 & 87.49 & 75.66 & -2.12 & 0.035 & * & 84.2 & 57.83 & -1.39 & 0.17 & \\
\hline IgA secretion rate $(\mu \mathrm{g} / \mathrm{min})$ & 23 & 54.94 & 42.87 & 42.42 & 37.12 & -2.40 & 0.017 & * & 44.05 & 41.5 & -2.13 & 0.035 & * \\
\hline
\end{tabular}

$\lg A$ and $S D$ indicated immunoglobulin $A$ and standard deviation, respectively

$Z$-score and $P$-values were calculated by Wilcoxon matched-pairs signed rank test (both tailed)

Participants with a lower baseline salivary secretion volume also had a lower baseline IgA concentration, and these values were further reduced after exercise (cluster A in Fig. 2). The clusters (clusters B and C) showed both a higher salivary secretion volume and IgA concentration at baseline. Based on these observations, we divided the individuals into two groups (cluster A vs clusters B $+C$ ), and evaluated the change in salivary parameters of each group. The Wilcoxon rank-sum test is used here. Cluster $\mathrm{B}+\mathrm{C}$ showed a significant decrease in IgA concentration after 3 weeks (Wilcoxon matched-pairs signed rank test, $Z=-2.12, P=0.034)$ and in IgA secretion after 6 weeks (Wilcoxon matched-pairs signed rank test, $\mathrm{Z}=-1.88, \quad P=0.021)$. The other comparisons showed no significant change. These data also indicated that the stimulated salivary flow rate and IgA secretion were positively correlated.

When individual characteristics were compared between clusters $\mathrm{A}$ and $\mathrm{B}+\mathrm{C}$, only body mass index (BMI) showed a significant difference $(P=0.039$; Table 2$)$. However, no significant difference was observed using adjusted $P$-values. The participant in cluster $\mathrm{A}$, who showed a lower salivary secretion rate and IgA concentration at baseline, had a higher BMI value of $23.4 \pm$ $2.8 \mathrm{~kg} / \mathrm{m}^{2}$, while those in clusters $B+C$ showed a lower BMI value $\left(20.5 \pm 3.4 \mathrm{~kg} / \mathrm{m}^{2}\right)$. The effect of disease and the use of medication on the salivary parameters were evaluated (Table 3). Stimulated saliva flow rate was affected by Parkinson's disease (The Wilcoxon rank-sum test, $Z=2.02, P=0.04$ ) and methyldopa (The Wilcoxon rank-sum test, $Z=2.02, P=0.04$ ), and the IgA concentration was affected antihypertensive agents (The Wilcoxon rank-sum test, $Z=2.03, P=0.04$ ). However, these significances disappeared using adjusted $P$-values calculated by FDR which controlled $\alpha$ error, by considering multiple independent univariate tests.

\section{Discussion}

\section{Comparison between our observation and other reports} We hypothesized that oral exercise for 6 weeks would contribute to improving immune system function, which induces increased saliva and IgA secretion. However, we did not observe these changes. This observation was inconsistent with previous studies that reported an increase in salivary IgA after moderate exercise in older people. For example, older people (ages 60 to 80 years) who participated in resistance training for 6 months showed a significant increase in salivary IgA, regardless their age or sex [27]. The daily activity levels of the older people in their 70s were classified using the number of steps, and participants with a higher number of steps secreted more saliva and IgA than those with fewer steps [5]. Resistance training and endurance training for 12 months in the older people (age 64.9 \pm 8.4 years) increased salivary IgA [28]. An oral functional exercise program showed that participants who had 20 or more remaining teeth showed an improvement in oral function after 6 months of exercise [23].
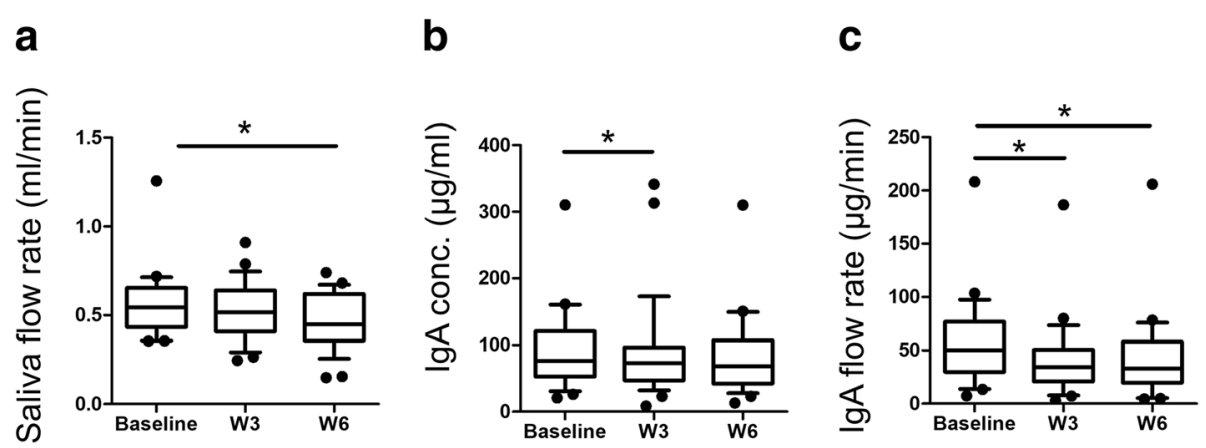

Fig. 1 Box-whisker plots of stimulated salivary secretion rate, concentration of IgA and its secretion rate. The horizontal bars indicate 10, 25, 50, 75 and $90 \%$ of the data, and data $<10 \%$ and $>90 \%$ are depicted in the plots. The data at baseline and at 3 and 6 weeks after oral functional exercise are labelled Baseline, W3 and W6. Saliva flow rate (a), IgA concentration (b), and IgA flow rate (c). Wilcoxon matched-pairs signed rank test (two-tailed) was used. ${ }^{*} P<0.05$ 


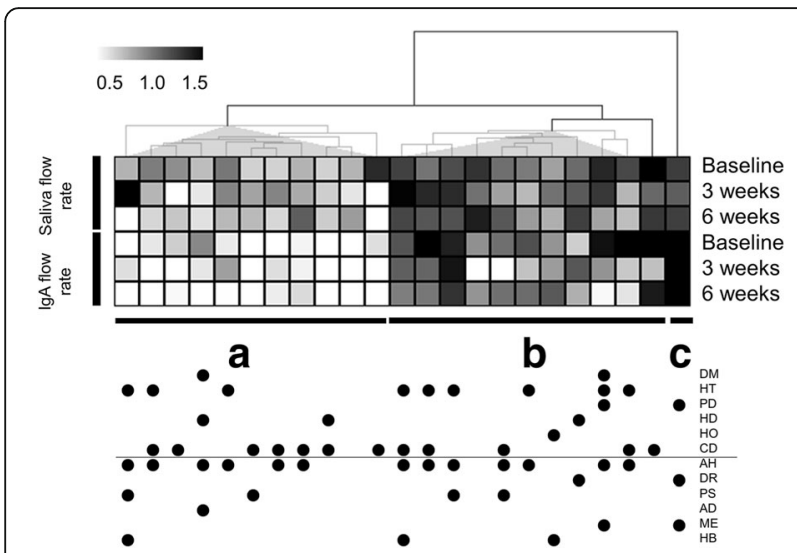

Fig. 2 The clustering results of participants based on the pattern of stimulated saliva flow rate and IgA secretion rate. The quantified values were divided by the averaged value of each sampling point and colored in black and white for relative higher and lower values, respectively, compared with their average. The color bar (at the upper left) indicates the fold change of each quantified values (0.5, 1.0 and 1.5 indicated fold change). Data at a line indicates the values collected at a time point and data at a column indicates the values for each subject. The top three lines and the bottom three lines indicate the rate of salivary secretion and IgA secretion rate data, respectively. Dendrogram (presented in tree form) indicates the similarity of each column, yielded by clustering analysis. Elucidation distance was used for the clustering algorithm, e.g. columns

showing similar data patterns were aligned closely. Diseases and the use of medication are represented by solid dots. Abbreviations are presented in Table 2. Prominent clusters are labelled $\mathbf{a}, \mathbf{b}$ and $\mathbf{c}$

There are three main differences between our study and other studies: (1) most of the participants in this study were older than the participants in other studies. All participants in our study were cared for in geriatric health services facilities and their daily activity level was expected to be lower than the participants who did not live in these facilities; (2) the duration of exercise was shorter than for other studies; and (3) whole body exercise was not used in our study, but rather the participants only performed oral functional exercises.

IgA concentration at younger ages (up to the 60s) increases, while it decreases in participants in their 60s; the IgA level in participants in their 60s was $92.9 \pm$ $6.42 \mu \mathrm{g} / \mathrm{ml} \mathrm{[24]}$. The baseline value in this study was $91.23 \pm 58.4 \mu \mathrm{g} / \mathrm{ml}$, which is consistent with other reports showing IgA concentration is lower in older people than in those in their 60s.

Oral functional exercise for 3 months in the older people (age, $77.9 \pm 6.5$ years) showed a drastic increase in oral lip closure and the amount of oral diadochokinesis. In addition, a repetitive saliva swallowing test also showed an increase in the amount of swallowing for the participants who had a lower amount of swallowing $(\leq 3)$, while the participants who showed more swallowing $(\geq 4)$ had no significant increase. It was concluded that the 3-month duration of the training was short and not enough to contribute to the recovery of their immune system [19]. A shorter duration of oral functional exercise in our study probably might not contribute to an improvement of the immune system. In addition, all salivary parameters were reduced after the training programs (Fig. 3), which was unexpected. Because both the immune system and acute stress contribute to increased IgA [20], a reduction in acute stress is one possible explanation while saliva samples were collected $1.5 \mathrm{~h}$ and after breakfast.

The observed salivary parameters, especially IgA concentration, showed large individual variances at baseline. One possible reason was the use of cotton for saliva collection, which may have caused the differences in salivary secretion; although small variations in IgA concentration resulting from differences in chewing forces have been reported [29]. Stress can also influence IgA concentrations [30]. Although salivary IgA is widely used to evaluate immune function, the profiling of other substance, such as, lactoferrine showing antimicrobial activity, would help identify subjects' specific patterns [31]. The use of simultaneous molecule profiling technique $[32,33]$ is also would contribute to understand the relationship between salivary parameters and factors that influence the parameters.

\section{Clustering analysis}

Clustering analyses were conducted to explore new subtype of the subjects based on the similarity of multiple observations. The analyses resulted in BMI was a primary parameters characterizing salivary parameters including both salivary flow rate and IgA concentrations (Fig. 2 and Table 2). Each cluster included subjects shows BMI value of $23.4 \pm 2.8$ and $20.5 \pm 3.4 \mathrm{~kg} / \mathrm{m}^{2}$. Both of them were not in overweight range. Among old women, overweight BMI was associated with slightly reduced mortality among those in poor health and underweight BMI was significantly associated with increased mortality [25]. Physical function was observed for the old person showing decline of BMI along with age [22]. These relationships between BMI and health conditions are considered as possible reasons for the association with BMI and immune functions, however, our observation should be confirmed by larger cohort to more confident conclusion.

\section{Conclusions}

There were some limitations to our study. Our participants showed large individual variances in salivary parameters; thus, enrolling a larger number of participants would be necessary to make stronger conclusions. More variables including salivary markers should be collected to identify the factors causing individual differences in salivary parameters. A clustering technique revealed that all participants were grouped into two groups based on 
Table 2 Comparison of participants' characteristics based on clustering analysis

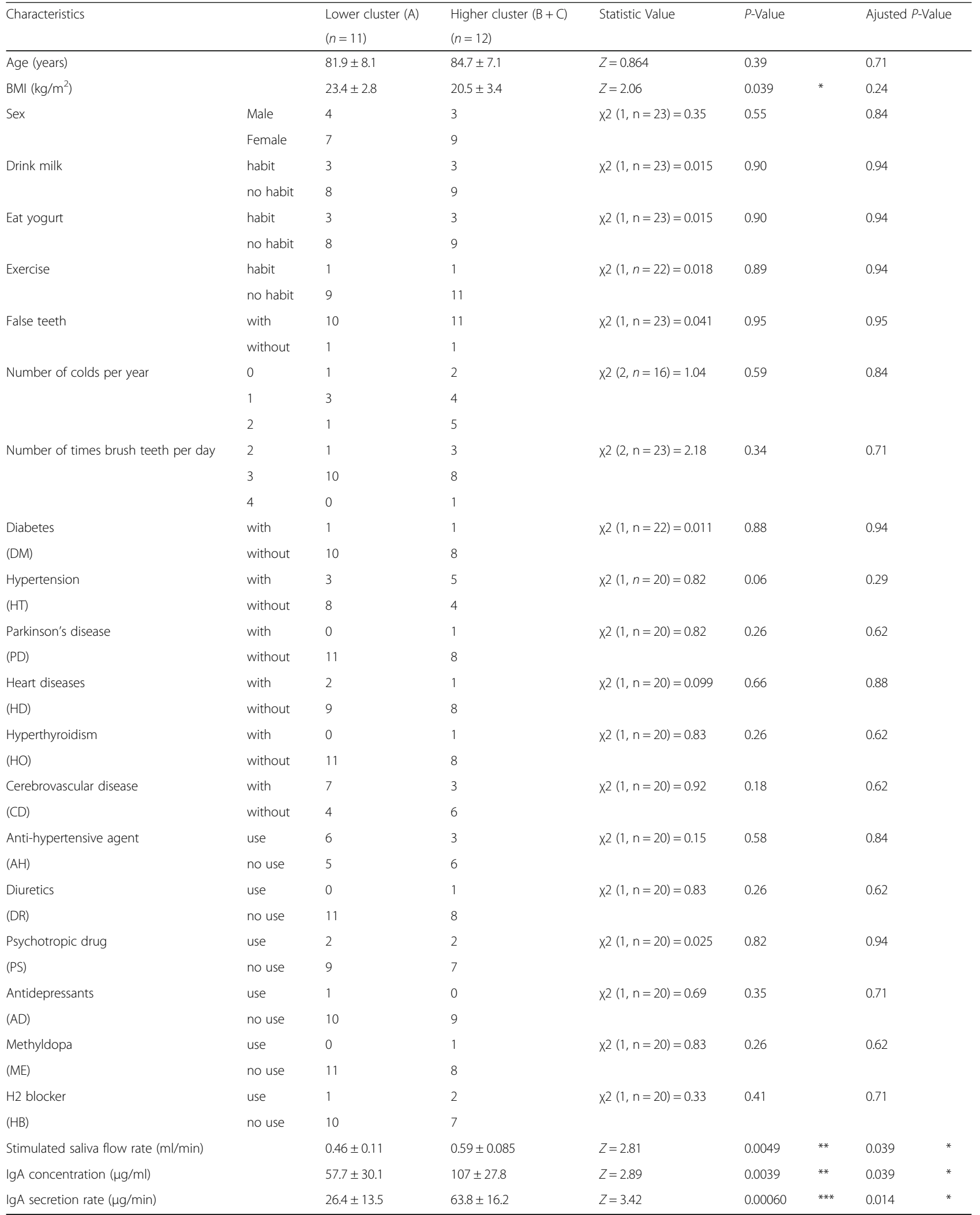

No participants had depression or epilepsy, and none used anti-hyperglycemic agents, antihistamine or a1-blocking drugs

Statistic value of $X 2$ test (two-sided) included degree of freedom, sample, size and $P$-values

$Z$-score and $P$-values were calculated by Wilcoxon rank-sum test (both tailed) 


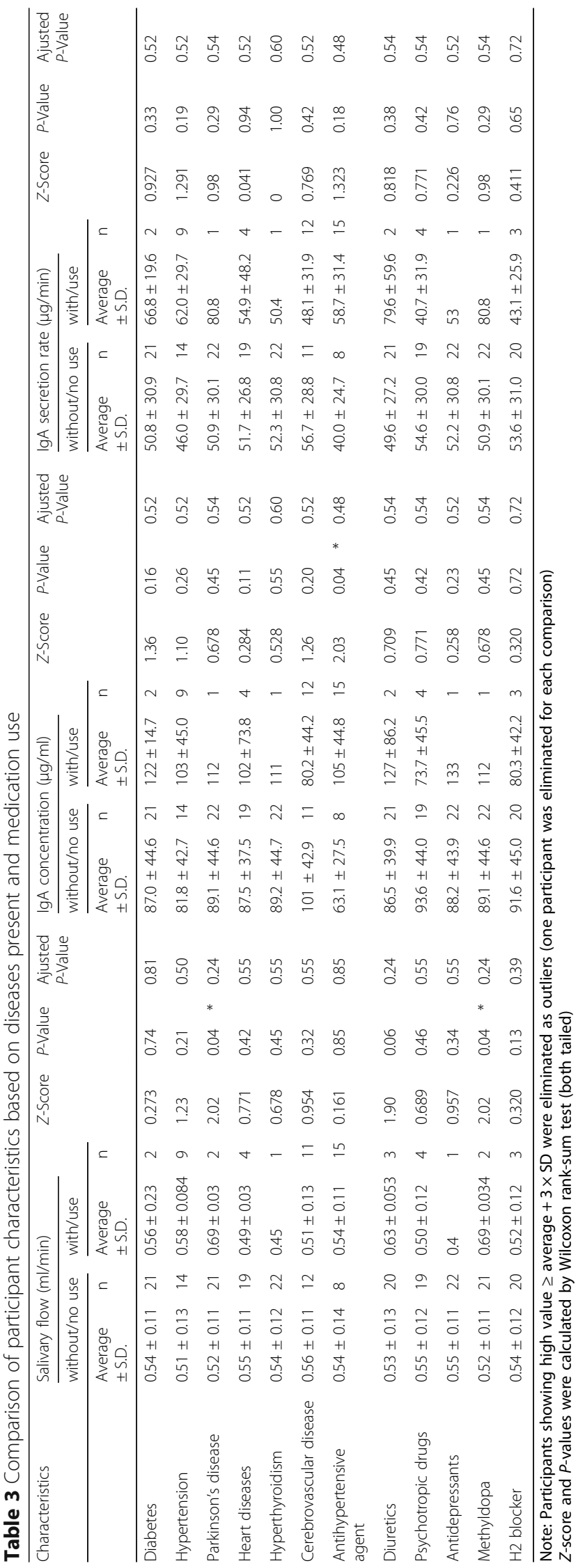



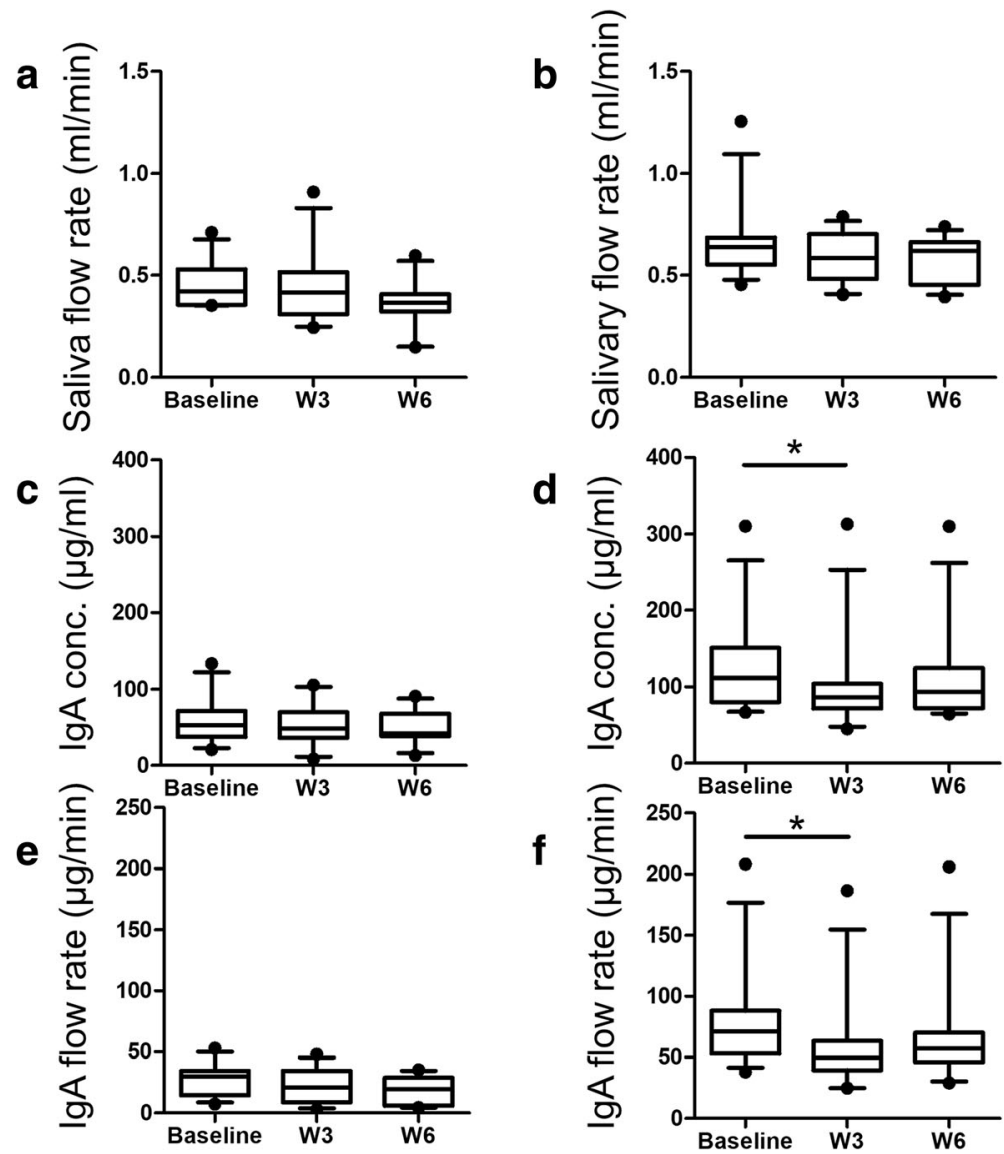

Fig. 3 The change in salivary parameters for each group showing a lower stimulated saliva secretion rate and IgA secretion rate (cluster $A$ in Fig. 2) and higher values (clusters $B+C$ in Fig. 2). Salivary secretion flow rate (a) and (b), concentration of IgA (c) and (d), and the secretion rate of $\lg \mathrm{A}(\mathbf{e})$ and $(\mathbf{f})$. Panels $\mathbf{a}, \mathbf{c}$ and $\mathbf{e}$ present the data in cluster $\mathrm{A}(n=11)$ and panels $\mathbf{b}, \mathbf{d}$ and $\mathbf{f}$ present the data in the clusters $\mathrm{B}+\mathrm{C}$ $(n=12)$. Only the data without any missing value was used. Wilcoxon matched-pairs signed rank test (two-tailed) was used. ${ }^{*} P<0.05$

the patterns of these salivary parameters. A significant change after oral functional exercise was observed only in the group showing a higher salivary secretion rate and IgA concentration at baseline. This is the first report to reveal such individually specific salivary parameter patterns in older people over the age of 80 years.

\section{Abbreviations}

BMI: body mass index; ELISA: enzyme-linked immunosorbent assay; FDR: false discovery rate; IgA: Immunoglobulin A; RSD: relative standard deviation; SD: standard deviation; URTI: upper-respiratory tract infections

\section{Acknowledgements}

The authors thank the study participants. We thank Ayame Enomoto and Kana Hiwatari at Keio University to help with the data analyses.

\section{Funding}

Not applicable.

\section{Availability of data and materials}

The datasets used and/or analysed during the current study available from the corresponding author on reasonable request.

\section{Authors' contributions}

MSato collected data. MSugimoto analysed data and wrote manuscript. YY measured data. MSato, JS, and KT designed this study. All authors read and approved the final manuscript.

\section{Authors' information}

MSato is a Professor at Tohto College of Health Sciences. MSugimoto is a Professor at Tokyo Medical University. YY is a Lecturer at Kanagawa Dental University. JS is an Associate Professor at Kanagawa Dental University. KT is a Professor at Kanagawa Dental University.

\section{Ethics approval and consent to participate}

This study was approved by the ethics committee at Ryotokuji University (No. 2506) and Oasis Geriatric Health Services facilities. The research was performed, following to the Declaration of Helsinki in 1995 (as revised in Brazil 2013). Informed consent was obtained from the volunteer participants and their families.

\section{Consent for publication}

Not applicable.

\section{Competing interests}

The authors declare that they have no competing interests.

\section{Publisher's Note}

Springer Nature remains neutral with regard to jurisdictional claims in published maps and institutional affiliations. 


\section{Author details}

'Nursing Science, Ryotokuji University, Urayasu, Chiba 279-8567, Japan. ${ }^{2}$ Nursing Science, Human Care Department, Tohto College of Health Sciences, Fukaya, Saitama 366-0052, Japan. ${ }^{3}$ Department of Oral Science, Graduate School of Dentistry, Kanagawa Dental University, Yokosuka, Kanagawa 238-8580, Japan. ${ }^{4}$ Institute for Advanced Biosciences, Keio University, Tsuruoka, Yamagata 997-0052, Japan. ${ }^{5}$ Health Promotion and Preemptive Medicine, Research and Development Center for Minimally Invasive Therapies, Tokyo Medical University, Shinjuku, Tokyo 160-0022, Japan. ${ }^{6}$ Department of Oral Science, Division of Salivary Gland and Health Medicine, Graduate School of Dentistry, Kanagawa Dental University, Yokosuka, Kanagawa 238-8580, Japan.

Received: 5 December 2016 Accepted: 14 December 2017

Published online: 08 January 2018

\section{References}

1. Roberts-Thomson IC, Whittingham S, Youngchaiyud U, Mackay IR. Ageing, immune response, and mortality. Lancet. 1974;2(7877):368-70.

2. Shephard RJ, Shek PN. Exercise, aging and immune function. Int J Sports Med. 1995;16(1):1-6

3. Nieman DC, Henson DA, Gusewitch G, Warren BJ, Dotson RC, Butterworth DE, Nehlsen-Cannarella SL. Physical activity and immune function in elderly women. Med Sci Sports Exerc. 1993;25(7):823-31.

4. Sakamoto $Y$, Ueki S, Kasai T, Takato J, Shimanuki H, Honda H, Ito T, Haga H. Effect of exercise, aging and functional capacity on acute secretory immunoglobulin a response in elderly people over 75 years of age. Geriatr Gerontol Int. 2009;9(1):81-8.

5. Shimizu K, Kimura F, Akimoto T, Akama T, Kuno S, Kono I. Effect of freeliving daily physical activity on salivary secretory IgA in elderly. Med Sci Sports Exerc. 2007:39(4):593-8.

6. Silva LC, de Araujo AL, Fernandes JR, Matias Mde S, Silva PR, Duarte AJ, Garcez Leme LE, Benard G. Moderate and intense exercise lifestyles attenuate the effects of aging on telomere length and the survival and composition of T cell subpopulations. Age. 2016:38(1):24

7. Woods JA, Ceddia MA, Wolters BW, Evans JK, Lu Q, McAuley E. Effects of 6 months of moderate aerobic exercise training on immune function in the elderly. Mech Ageing Dev. 1999;109(1):1-19.

8. Chen X, Kistler CE. Oral health care for older adults with serious illness: when and how? J Am Geriatr Soc. 2015;63(2):375-8.

9. Petersen PE, Yamamoto T. Improving the oral health of older people: the approach of the $\mathrm{WHO}$ global oral health Programme. Community Dent Oral Epidemiol. 2005;33(2):81-92.

10. Azarpazhooh A, Leake JL. Systematic review of the association between respiratory diseases and oral health. J Periodontol. 2006;77(9):1465-82.

11. Persson R. The significance of periodontal inflammation on systemic health in old age. Gerodontology. 2012;29(3):169-70.

12. Yoneyama $T$, Hashimoto $K$, Fukuda $H$, Ishida M, Arai H, Sekizawa K, Yamaya M, Sasaki H. Oral hygiene reduces respiratory infections in elderly bedbound nursing home patients. Arch Gerontol Geriatr. 1996;22(1):11-9.

13. Migliorati $C$, Madrid C. The interface between oral and systemic health: the need for more collaboration. Clin Microbiol Infect. 2007;13(s4):11-6.

14. Sumi $Y$, Ozawa N, Miura H, Michiwaki Y, Umemura O. Oral care help to maintain nutritional status in frail older people. Arch Gerontol Geriatr. 2010; 51(2):125-8.

15. Park K, Choi-Kwon S, Park M-S. The effects of oral health care education for nursing staff on halitosis and nutrient intake of the elderly in a long-term care facility. Journal of the Korean Dietetic Association. 2011;17(3):243-58.

16. Dahm TS, Bruhn A, LeMaster M. Oral Care in the Long-Term Care of older patients: how can the dental hygienist meet the need? American Dental Hygienists Association. 2015;89(4):229-37.

17. Duffy JR. Motor speech disorders: substrates, differential diagnosis, and management. Elsevier Health Sciences. 2013;

18. Kikutani T, Enomoto R, Tamura F, Oyaizu K, Suzuki A, Inaba S. Effects of oral functional training for nutritional improvement in Japanese older people requiring long-term care. Gerodontology. 2006;23(2):93-8.

19. Ooka T, Haino T, Hironaka S, Mukai Y. The effect of daily oral function training in the elderly. J Dent Health. 2008:58:88-94. (in Japanese)

20. Sugiyama T, Ohkubo M, Honda $Y$, Tasaka A, Nagasawa $K$, Ishida R, Sakurai K. Effect of swallowing exercises in independent elderly. The Bulletin of Tokyo Dental College. 2013;54(2):109-15.
21. Tenovuo JO: Human saliva, vol. 1: CRC press; 1989

22. Reinders I, Murphy RA, Martin KR, Brouwer IA, Visser M, White DK, Newman $A B$, Houston DK, Kanaya AM, Nagin DS, et al. Body mass index trajectories in relation to change in lean mass and physical function: the health, aging and body composition study. J Am Geriatr Soc. 2015;63(8):1615-21.

23. Diehr P, O'Meara ES, Fitzpatrick A, Newman AB, Kuller L, Burke G. Weight, mortality, years of healthy life, and active life expectancy in older adults. J Am Geriatr Soc. 2008:56(1):76-83.

24. Jafarzadeh A, Sadeghi M, Karam GA, Vazirinejad R. Salivary IgA and IgE levels in healthy subjects: relation to age and gender. Brazilian oral research. 2010; 24(1):21-7.

25. Leigh $\mathrm{L}$, Byles JE, Jagger $\mathrm{C}$. BMI and healthy life expectancy in old and very old women. Br J Nutr. 2016:1-8.

26. Saeed Al, Sharov V, White J, Li J, Liang W, Bhagabati N, Braisted J, Klapa M, Currier T, Thiagarajan M, et al. TM4: a free, open-source system for microarray data management and analysis. BioTechniques. 2003;34(2):374-8.

27. Shimizu K, Kimura F, Akimoto T, Akama T, Otsuki T, Nishijima T, Kuno S, Kono I. Effects of exercise, age and gender on salivary secretory immunoglobulin a in elderly individuals. Exerc Immunol Rev. 2007;13:55-66.

28. Akimoto T, Kumai Y, Akama T, Hayashi E, Murakami H, Soma R, Kuno S, Kono I. Effects of 12 months of exercise training on salivary secretory IgA levels in elderly subjects. Br J Sports Med. 2003;37(1):76-9.

29. Soeda R, Tasaka A, Sakurai K. Influence of chewing force on salivary stress markers as indicator of mental stress. J Oral Rehabil. 2012:39(4):261-9.

30. Takatsuji K, Sugimoto Y, Ishizaki S, Ozaki Y, Matsuyama E, Yamaguchi Y. The effects of examination stress on salivary cortisol, immunoglobulin a, and chromogranin a in nursing students. Biomed Res. 2008;29(4):221-4.

31. Ide M, Saruta J, To M, Yamamoto Y, Sugimoto M, Fuchida S, Yokoyama M, Kimoto S, Tsukinoki K. Relationship between salivary immunoglobulin a, lactoferrin and lysozyme flow rates and lifestyle factors in Japanese children: a cross-sectional study. Acta Odontol Scand. 2016;74(7):576-83.

32. Neyraud E, Tremblay-Franco M, Gregoire S, Berdeaux O, Canlet C. Relationships between the metabolome and the fatty acid composition of human saliva; effects of stimulation. Metabolomics. 2013;9(1):213-22.

33. Sugimoto M, Saruta J, Matsuki C, To M, Onuma H, Kaneko M, Soga T, Tomita M, Tsukinoki K. Physiological and environmental parameters associated with mass spectrometry-based salivary metabolomic profiles. Metabolomics. 2013;9(2):454-63.

\section{Submit your next manuscript to BioMed Central and we will help you at every step:}

- We accept pre-submission inquiries

- Our selector tool helps you to find the most relevant journal

- We provide round the clock customer support

- Convenient online submission

- Thorough peer review

- Inclusion in PubMed and all major indexing services

- Maximum visibility for your research

Submit your manuscript at www.biomedcentral.com/submit
) Biomed Central 